\title{
Optimization Analysis of Mobile Phone Shell Based on Moldflow
}

\author{
BaofuSun ${ }^{1}$,a , Mingjiang Hu ${ }^{1, b}$, Haixiong Wang ${ }^{1, c}$ \\ ${ }^{1}$ Add: 12\#Jian GanRd.Guilin Guangxi China \\ a1204605467@qq.com, b854288345@qq.com, c188169662@qq.com
}

\begin{abstract}
Keywords: mobile phone shell; moldflow; Taguchi; optimizing design
Abstract. For simple plastic injection parts of mobile phone shell, in order to reduce the warp of the injection molding and the number of test mold in design process, this paper takes a mobile phone shell for example as the analysis object, using SolidWorks software to address the Three-dimensional modeling of the mobile phone shell and moldflow software to analyze the warp defects of product .By the way of taking the method of Taguchi experiment, optimizing mold structure, filling pressure and sizing pressure curve,the warp of the injection molding could be reduced. So we can get a better combination data of process and reduce the cost of mold-design and manufacturing.
\end{abstract}

\section{Introduction}

The plastic mold industry plays an important part in national manufacturing industry, especially the injection mold in prevalent applying of plastic products at this time. In recent years, lots of electronic products like mobile phones are updating rapidly and better but cheaper shells of mobile phones are needed by users. Therefore, the applying of injection mould production can meet the market requirements for such mobile phones because it can be produced on a large scale at a lower price. Nevertheless, the designing and producing period of such mobile phones is obviously too long as a result of the repeating mold-testing and mold-fixing features that traditional designing and manufacturing technology holds. In order to adapt to the rapid updating of a mobile phone shell, the software Moldflow can help to resolve problems that exist in the process of the mold structure and formation in advance. The designing quality of The mold could be improved efficiently and the producing period as well as its cost of production could be reduced because the designing defects will be optimized or removed before the mold has been manufactured.

What this paper is going to analyzing is a mobile phone's shell which has a simple design together with a snap joint, veins and holes around the side shell. As the mobile phone's shell can be produced in large volumes, this paper makes an analysis of the formation process of injecting plastic by using the moldflow software and finds the reason why the plastic gets twisted in injecting plastic process. As it has been optimized to some extend, the success rate of mold-testing will be increased and its cost of production and manufacturing period will be reduced.

\section{Pretreatment Analysis}

Use the SolidWorks software to address the three-dimensional model of the mobile phone shell, the maximum size of the length direction of the three-dimensional is $130 \mathrm{~mm}$, the maximum width of the direction is $66 \mathrm{~mm}$, the height direction is $6 \mathrm{~mm}$.Export IGES file after completion of the model,Importthe softwareof Autodesk simulation design link, not automatically generate the grid,conversion the surface, and finally generate the grid. After repaired by CADdoctor, the mesh quality of matching percentage is $95.3 \%$, the percentage of each other is $93.8 \%$, the maximum aspect ratio is 6.6, which meets the requirement of warping analysis.According to the characteristics of the mobile phone shell, the mold should be selected the submarine gate orside gate,Use the control method of the constraint node to select the gate position. After the best gate location and rapid filling analysis, according to the influence of weld line[1] and filling pressure[2] on the forming of products, selection scheme of two gate . 


\section{Analysis Arrangement Fill Analysis}

The Moldflow library provides materials to promote process parameters. According to referral parameters, setting the mold surface temperature is 60 DEG C, the melt temperature is 230 DEG C, using automatic filling control mode, speed pressure switch adopts automatic mode, The pressure control adopts $80 \%$ filling pressure, the time is 10 seconds. Filling results as shown that the maximum pressure of the cavity is $34.68 \mathrm{MPa}$, less than $40 \mathrm{MPa}$, the filling time is $0.6766 \mathrm{~s}$, according to the data obtained from the molding window is 0.66 seconds, error less than 0.5 seconds . The maximum pressure of the $\mathrm{v} / \mathrm{p}$ switch is $37.27 \mathrm{MPa}$, and the pressure value is low, which is favorable for the injection molding machine. The temperature range of the flow front is 227.9 DEG C 232 DEG C, which is in the range of the melt temperature, and the temperature range from the proposed temperature is less than or equal to 2 degrees, which is less than the empirical value 20 degrees, and no short shot, conducive to forming.

Cooling + Filling + Pressure + Warpage Analysis. According to the filling results choice the analysis sequence is "cooling + filling + holding pressure + warping", the surface temperature of the mold is $60 \mathrm{DEG} \mathrm{C}$, the melt temperature is $230 \mathrm{DEG} \mathrm{C}$, the time of the "injection + pressure maintaining + cooling" is set to 30 seconds, open time is set to 5 seconds, the injection time is set to 0.72 seconds, speed pressure switch is adot automatic mode, the filling pressure of the pressure control adopts $80 \%$, the time is 10 seconds, Separation the reason of warp. According to the volume shrinkage path graph we can see that the difference between the maximum and the minimum of the volume shrinkage rate is more than $3 \%$ of the recommended ABS. The temperature of the cooling circuit medium is less than 2 DEG $\mathrm{C}$, and the change temperature of the pipe wall is less than 5 DEG $\mathrm{C}$, and the design of the water channel is basically reasonable. The deformation of all effects is $0.556-0.1450=0.411 \mathrm{~mm}$, the deformation caused by the uneven cooling is $0.041 \mathrm{~mm}$, the deformation caused by shrinkage deformation amount is $0.5454-0.1446=0.4008$, due to the adoption of ABS did not add fiber materials, so the deformation caused by orientation is 0 .

\section{Optimal Design}

Moldflow Taguchi Experiment. According to the results of the initial analysis, the optimization experiment is established.Moldflow software was used to establish the experiment of Taguchi variables, the melt temperature, injection time, holding time, filling pressure, injection + holding pressure + cooling time 5 variables as variables information, the number is 8 times. The percentage of deformation as a result,the obtained experimental results are expressed in standard deviation,summarized as shown in Table 1.

Table 1 Effect of global weighted mass

\begin{tabular}{cccccc}
\hline Influence factor & $\begin{array}{c}\text { MeltTem } \\
\text { perature }\end{array}$ & $\begin{array}{c}\text { Injectionti } \\
\text { me }\end{array}$ & $\begin{array}{c}\text { Holding } \\
\text { time }\end{array}$ & $\begin{array}{c}\text { Filling } \\
\text { pressure }\end{array}$ & $\begin{array}{c}\text { Injection + pressure + } \\
\text { cooling time }\end{array}$ \\
\hline $\begin{array}{c}\text { Quality } \\
\text { (deformation) }\end{array}$ & $12.13 \%$ & $20.94 \%$ & $1.25 \%$ & $50.92 \%$ & $14.76 \%$ \\
\hline
\end{tabular}

According to the influence of the weighted mass, it can be seen that the influence factors of the deformation coefficient of the mobile phone shell are the filling pressure, injection time, injection pressure, holding pressure, cooling time, melt temperature and holding time.

Optimizing Filling Pressure. Mobile phone shell is thin-walled part, the main deformation is warpage[3],we adopt the pressure control method with filling pressure and time, which set up 80\%, $100 \%, 120 \%, 140 \%$ filling pressure. The results show that the larger the filling pressure is, the smaller the volume shrinkage is, when the filling pressure is $120 \%$, the volume shrinkage is $3.827+0.9055=4.7325$. As we can be seen in Figure 1, the greater the filling pressure, the smaller the deformation, When the filling pressure is $80 \%$, the deformation is $0.5173-0.1367=0.3806 \mathrm{~mm}$, When the filling pressure is $140 \%$, the deformation of all effects is $0.2667-0.0137=0.253 \mathrm{~mm}$, and the deformation decreased by $33.5 \%$, But too much pressure will increase the clamping force, increase 
energy consumption, count against to enterprise production. According to the residual stress in the first direction of the cavity, the volume shrinkage rate at the top, the deformation and maximum clamping force of Injection molding machine etc chose the filling pressure as $120 \%$ of the default filling pressure,optimizing the holding pressure curve to Improve the quality of injection mold parts.

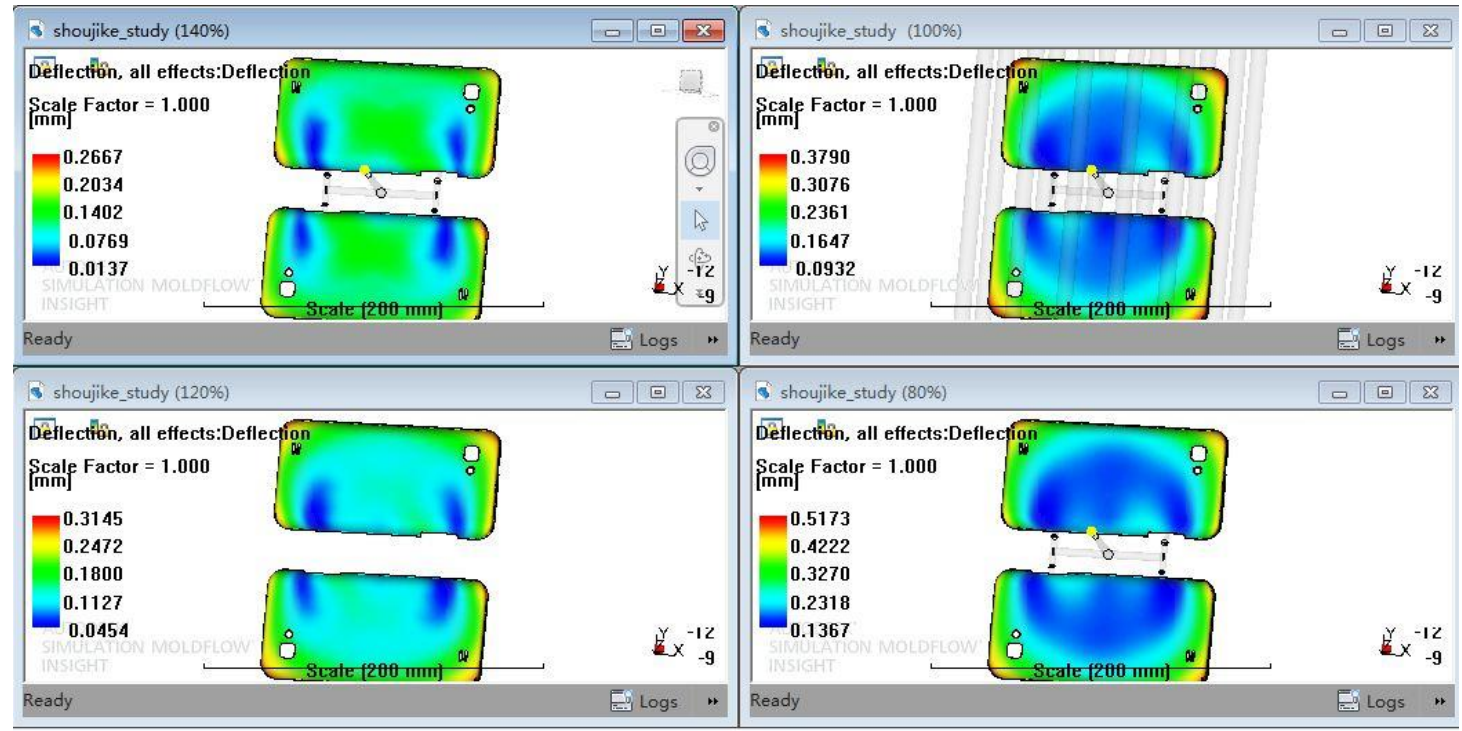

Figure 1. all effects: dellection

Optimize the Curve of Pressure. The curves of pressure and time of XY diagram can be used to predict the volume shrinkage of injection molded parts, the corresponding curves formed by the closer, the more stable the volume shrinkage, injection molding product quality is better, It can be used as a reference for optimizing the pressure maintaining curve.According to the principle of optimization, the time of constant pressure stage is chosen to fill the end pressure,XY diagram for reference,maximum pressure time is 1.209 seconds,pressure to zero for 3.463 seconds, the middle point of time is $(1.209+3.463) / 2=2.336$ seconds,. The time of the constant pressure is V/P minus 0.786 of the time point, so constant pressure time is $2.336-0.786=1.55$ seconds. The time of holding pressure attenuation is determined according to the freezing layer factor[4]. Time is $5.58-2.336=3.244$ seconds, draw the pressure curve. After optimization, the change of the volume shrinkage rate is within 3\%, which is consistent with the shrinkage of ABS. Figure 2 is to optimize the deformation after the pressure curve, the constant pressure deformation is $0.3145-0.0454=0.2691 \mathrm{~mm}$, the amount of deformation obtained by the method of attenuating the pressure is $0.2998-0.0485=0.2513 \mathrm{~mm}$. The amount of warpage relative to constant pressure continue to reduce the pressure of $6.7 \%$, injection molding products have basically meet the demand. 


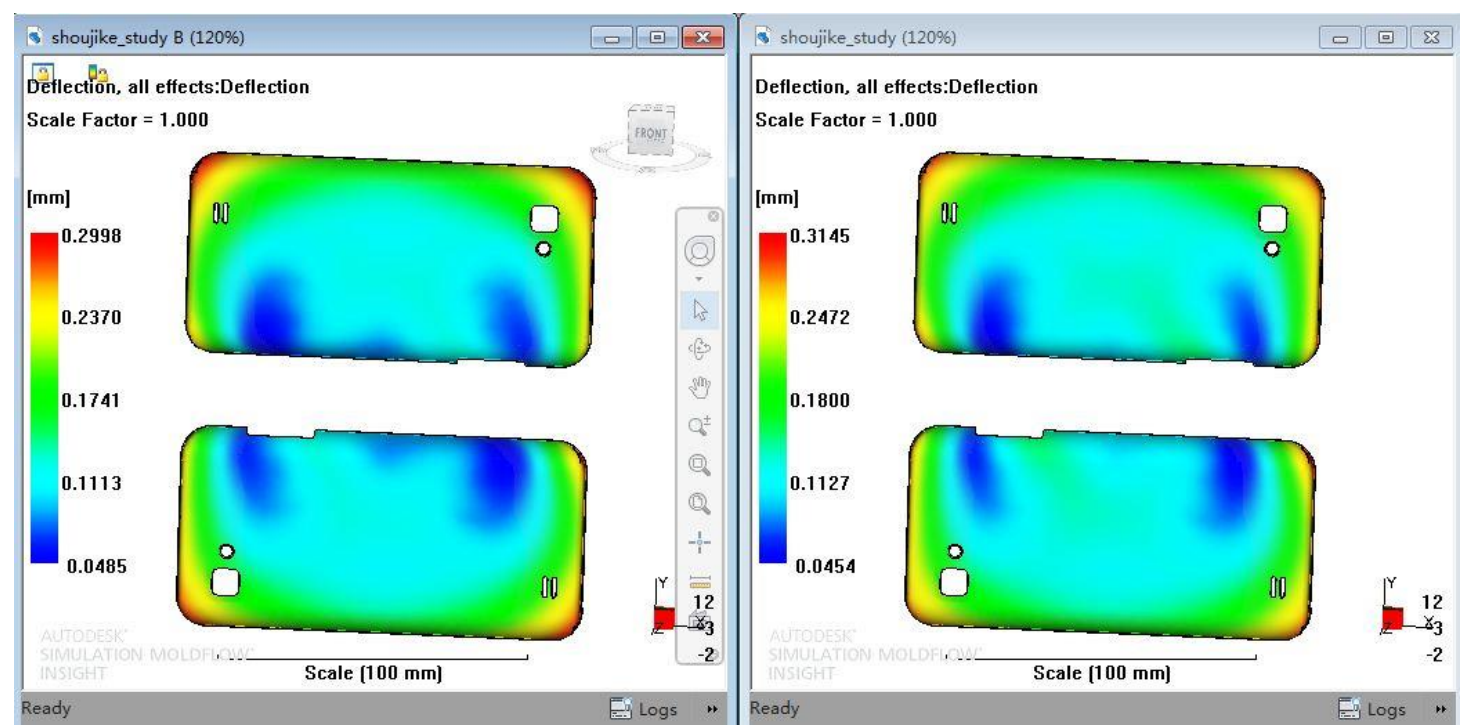

Figure 2. Optimization of all effects : dellection

\section{Conclusion}

The taguchi experimental method can quickly determine the factors affecting deformation based on Moldflow software,the influence level of the quality is from large to small, respectivelyas follows, the filling pressure, injection time, injection + holding pressure+cooling time, melt temperature and holding time.The XY diagram of pressure at the end of filling can provide reference for optimizing the pressure maintaining curve and improving the warpage of injection molded parts.

\section{Reference}

[1] YunSun, QianWang. Optimization the weld defects of mobile phone shell based on Moldflow [A]. Light Industry Science and Technology,2015,9:68-69.In Chinese

[2] YediChen. Optimization the gate location of battery box based on CAE [J]. Plastics Science and Technology 2012.40 (6): 61-64. In Chinese

[3] Xuan-phuong Dang. General frameworks for optimization of plastic injection molding process parameters [J]. Simulation Modelling Practiceand Theory,2014( 41) : 15-27.

[4] DeqiangZhang, YanZhang, XiaoxinMeng, JinhuaLi. Pressure curve optimization method research based on Moldflow [A]. Journal of Liaoning University of Technology (JCR Science Edition), 2015.35 (1): 38-39. In Chinese 